\title{
Microstructure and Mechanical Properties of Copper Billets Fabricated by the Repetitive Extrusion and Free Forging Process
}

Yang Wu, Shuhai Huang, Qiang Chen, Bo Feng, Dayu Shu, and Zhiwei Huang

\author{
(Submitted August 10, 2018; in revised form January 10, 2019; published online February 14, 2019)
}

\begin{abstract}
In the present study, with the aim of obtaining a weakened texture, grain refinement and improved mechanical properties, copper billets were successfully fabricated using the severe plastic deformation method of repetitive extrusion and free forging (REFF) without using any additional tools or devices. The evolution of the microstructure, texture and mechanical behavior of the ultrafine grain copper billets over an increasing number of REEF cycles was also systematically studied. Our results show that the effective strain per REFF process was $\varepsilon=\mathbf{2 . 3 5}$. After two cycles of REFF process, the sum effective strain became $\varepsilon=4.7$, leading to high efficiency of grain refinement. The grain size was refined from 50 to $0.8 \mu \mathrm{m}$; the yield strength was increased to $462 \mathrm{MPa}$, and we obtained a weakened texture in the pure copper billets. By increasing the number of cycles, the grain refinement ability was decreased, and the texture was thus strengthened further. Therefore, two cycles of REFF were a reasonable process for producing ultrafinegrained copper billets.
\end{abstract}

Keywords microstructure, pure copper, repetitive extrusion and free forging, texture

\section{Introduction}

Pure copper and its alloys with the high electrical and thermal conductivity, high formability, machinability and relatively moderate properties are increasingly being concerned in many industries (Ref 1-3). However, pure copper ingots, prepared by casting, are composed primarily of coarse-grained (CG) microstructures (Ref 4). For some applications, these microstructures are not a problem because these applications do not depend on isotropic or superior mechanical properties, but for particular applications, it is important for the $\mathrm{Cu}$ to have ultrafine-grained (UFG) microstructures and extremely uniform mechanical properties. For instance, some of these applications include use as thin sheets for co-deformation with pure $\mathrm{Cu}$ for $\mathrm{Nb}_{3} \mathrm{Sn}$ superconductor diffusion barriers or as thick sheet disks for explosively formed penetrators (Ref 5). Without uniformity of microstructure, a weakened texture and a grain refinement microstructure, the characteristics of uniform deformation are absent and problems such as orange peel surface roughness, non-uniform thinning and premature facture can arise (Ref 6-8).

Ultrafine-grained materials have spawned much interest in fields where extremely high tensile and yield strength are necessary for construction, as an alternative to coarse-grained

Yang Wu, Shuhai Huang, Qiang Chen, Dayu Shu, and Zhiwei Huang, Southwest Technology and Engineering Research Institute, Chongqing 400039, China; and Bo Feng, Guangdong Institute of Materials and Processing, Guangdong Academy of Sciences, Guangzhou 510650, China. Contact e-mail: 2009chenqiang@hfut.edu.cn.
(CG) counterparts for satisfactory ductility (Ref 4, 9). A commonly used set of methods for fabricating UFG materials is the severe plastic deformation (SPD) methods, of which the best-known SPD techniques for producing UFG materials are the equal-channel angular pressing (ECAP) and the highpressure torsion (HPT) (Ref 10-14). Various UFG SPD materials (e.g., Cu (Ref 15, 16), Al (Ref 17), Ta (Ref 18), Ti (Ref 19) and steel (Ref 6)) have been prepared using ECAP or HPT processing. Microstructures can be refined to equiaxed subgrains, which is accompanied by a significant increase with a satisfactory ductility. However, materials produced by ECAP and HPT have a small size along the transverse direction (Ref 20). This can limit the industrial applications of these methods for producing bulk billets for post-forging.

Extrusion and forging processes are suitable for preparing UFG materials at room or slightly above. It is well known that extrusion and free forging processes are highly efficient and simple, making them ideal for industrial applications. Therefore, we propose to combine these two traditional processes in cyclic processes of conventional direct extrusion and by free forging, a process termed repetitive extrusion and free forging (REFF). Compared with other SPD methods, REFF has several advantages, such as a higher strain per cycle, more shear planes and a more effective grain fragmentation. A similar approach, repetitive extrusion and upsetting (REU), has been reported by some publications (Ref 21). One publication reported the application of the REU process for fabricating pure aluminum billets with only $20 \mathrm{~mm}$ length along the longitudinal direction and $30 \mathrm{~mm}$ along the transverse direction. Unfortunately, this size of REU billets was insufficient to meet the needs of industrial applications. However, compared with REU, REFF has several advantages of a higher strain per cycle, a lower working force and no need for any additional tools or devices. Thus, REFF processing should be suitable for efficient and cost-saving industrial applications. In the present study, bulk size pure copper billets with excellent grain refinement microstructure for industry application, $80 \mathrm{~mm}$ in length along 
the longitudinal direction and $90 \mathrm{~mm}$ along the transverse direction, were successfully fabricated from coarse-grained pure $\mathrm{Cu}$. Three cyclic REFF passes were achieved in this work. The microstructure, texture evolution and mechanical properties of the extrusion and forging of pure $\mathrm{Cu}$ from each REFF cycle pass were also systematically studied.

\section{Experiments and Methods}

\subsection{REFF Process and Mechanical Tests}

Commercially pure copper ingots containing coarse-grained (CG) counterparts and fully annealed condition were used for the fabrication of the UFG copper billets. As shown in Fig. 1, the CG pure copper was machined into a cylinder with $90 \mathrm{~mm}$ in diameter and $80 \mathrm{~mm}$ in length. This ingot was kept in a furnace at $660{ }^{\circ} \mathrm{C}$ for $2 \mathrm{~h}$ and then cooled at room temperature in order to obtain a complete recrystallized microstructure. Before testing, the billets were covered with lubricant zinc stearate and then were extruded at room temperature using an extrusion rate of $10 \mathrm{~mm} / \mathrm{s}$. The final extruded bar had a diameter of $50 \mathrm{~mm}$. After complete extrusion, the extruded bar was free forged at the same rate until the initial diameter $(90 \mathrm{~mm})$ was reached again, so that the extrusion process could be repeated. For tensile tests, the dog-bone-shaped specimens with a gauge length of $35 \mathrm{~mm}$ and a diameter of $5 \mathrm{~mm}$ were cut along the longitudinal direction (ED or FD). Here, ED and FD refer to the extrusion direction and free forging direction, respectively. Tensile tests were performed on a Shimadzu mechanical testing system, using a strain rate of $10^{-3} / \mathrm{s}$ at room temperature. Each mechanical test was repeated three times.

\subsection{Characterization of Microstructure and Texture}

For studying the structural evolution, samples were cut from the center of the specimen perpendicular to the compression plane and parallel to the ED and/or FD. The obtained surface was polished and subsequently etched. Electron backscattered diffraction (EBSD) analyses were conducted on a FEI Nova 400 SEM equipped with an HKL Chanel 5 system, using a step size of $1 \mu \mathrm{m}$ for the initial $\mathrm{CG} \mathrm{Cu}$ material and the first extrusion billets, $0.3 \mu \mathrm{m}$ for the first forging billet and the second extrusion billets and $0.15 \mu \mathrm{m}$ for the second forging billets. Each specimen was scanned at three different areas to measure the average grain size. The samples for EBSD mapping were mechanically ground, followed by electrochemical polishing for $7 \mathrm{~s}$ at $273 \mathrm{~K}$ in the electrolyte solution of $50 \% \mathrm{H}_{3} \mathrm{PO}_{4}, 25 \%$ distilled water and $25 \%$ alcohol at a DC voltage of $6.5 \mathrm{~V}$. The EBSD data were analyzed using the Channel 5 software. Grain boundaries were identified using $2^{\circ}$ minimum disorientation angle between two adjacent pixels, which led to the identification of grains having continuous closed boundaries with at least a $2^{\circ}$ grain boundary angle. Grain orientations were then defined as the average of the pixel orientations constituting the grain, and then misorientation angle between adjacent grains was obtained using these average values (Ref 22).

\section{Results and Discussion}

\subsection{Repetitive Extrusion and Free Forging Process}

The present study shows that ultrafine-grained copper billet with bulk size for industry applications could be successfully

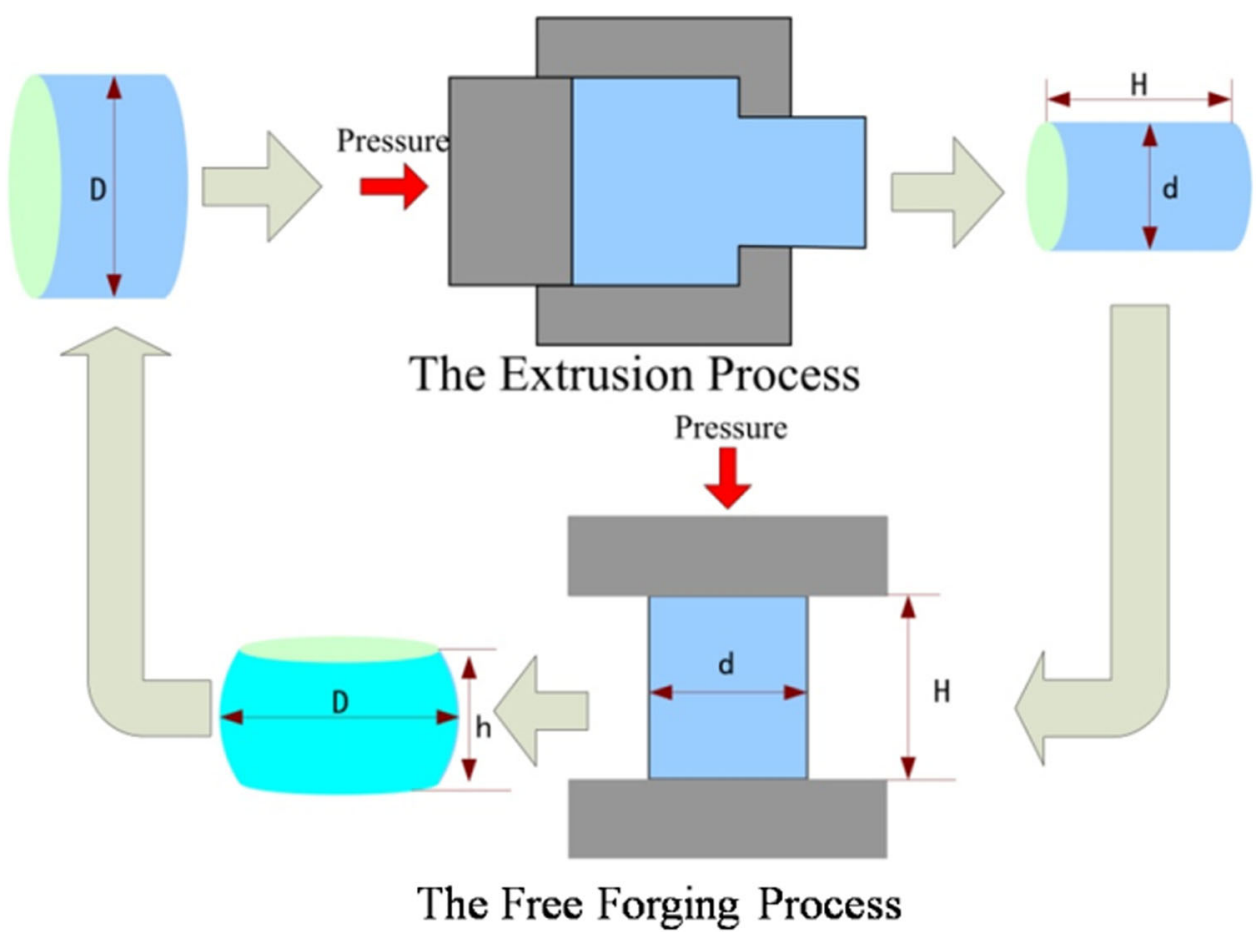

Fig. 1 Schematic diagrams of the cyclic REFF process 
fabricated from $\mathrm{CG} \mathrm{Cu}$ ingots using the REFF process. During each cycle of the REFF process, a high effective strain and an average von Mises strain from the geometric dimensions of the die using the normal components of the strain tensor were obtained, as defined below (Ref 21$)$ :

$\varepsilon=4 \ln \frac{D}{d}$

For the extrusion process, the diameter of the initial CG copper ingot and the extruded UFG copper was replaced by $D$ $(90 \mathrm{~mm})$ and $d(50 \mathrm{~mm})$, as shown in Fig. 1, respectively. Therefore, the extrusion ratio was 3.24 , and the corresponding effective strain per REFF process was $\varepsilon=2.35$. After two cycles, the sum effective strain became $\varepsilon=4.7$. Because the strain value in the center position was very close to the strain value of calculation, and it generally increases from the center to the surface of the $\mathrm{Cu}$ billet, so the microstructure of the billet surface was preferentially refined (Ref 22). In order to obtain whether a homogeneous microstructure from the center to the surface of the billet had been obtained, samples for mechanical and microstructure testing were cut from the center of each billet, parallel to the extrusion or/and forging direction, respectively.

\subsection{Microstructure and Texture Evolution During the REFF Process}

A fully annealed grain microstructure, as seen in the sample before REFF processing, is shown in Fig. 2. This structure includes a high fraction of annealing twins in the copper billet. The grains of CG copper were nearly equiaxed, and their average size was found to be about $50 \mu \mathrm{m}$, excluding twin boundaries. This was typical of a $\mathrm{Cu}$ microstructure after recrystallization. Before the REFF process, an equiaxed grain microstructure was obtained using fully annealing, in order to eliminate strain hardening and texture from previous metal working processes.

An EBSD analysis of the microstructure, crystallographic orientation maps and the misorientation angle distribution of the pure copper fabricated by the REFF process are shown in Fig. 3 and 4, respectively. The microstructures of the first extrusion and the first forging samples are displayed in Fig. 3(a) and (b), respectively. As seen in Fig. 3(a), an obvious elongation along the extrusion direction microstructure was

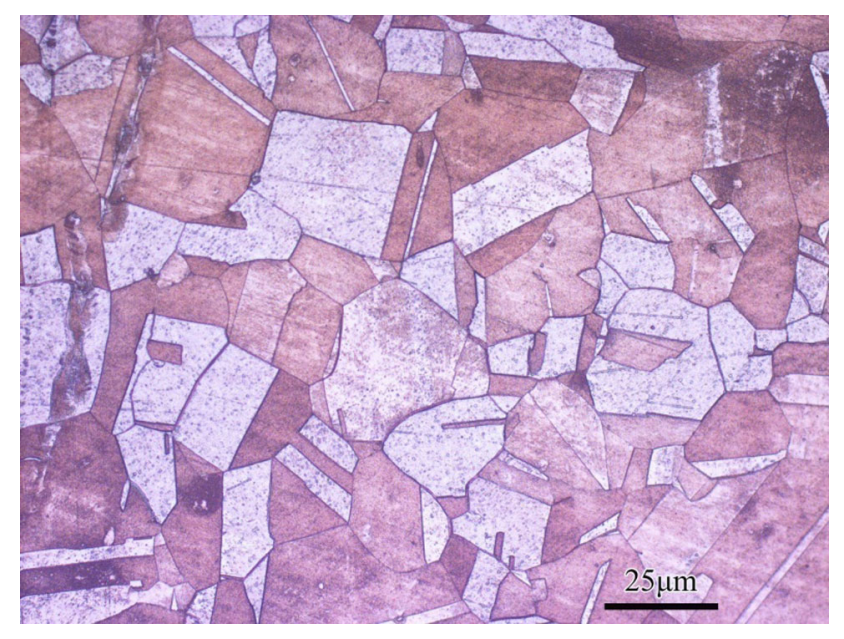

Fig. 2 Optical microstructures of the fully annealed CG copper obtained, so the initial annealed structure (Fig. 2a) gradually evolves into a pancake-like structure during the extrusion process. The edge dislocations and twin boundaries could not obviously be discerned from the elongation grains. However, following the subsequent forging step, these elongated grains transformed back into an equiaxed structure (Fig. 3b), which was different from the initial CG structure because of the large strain. During per cyclic REFF process, the strain path began with extrusion, and then it continued during forging, so the direction as well as the amount of strain was inverted and the grains recovered their initial shape. The strain-induced parallel dislocation walls and twin boundaries, formed by accumulation and rearrangement of dislocations and fragmentation of grains during the REFF process, are clearly shown in Fig. 3 and 4.

After the first extrusion process, a large number of lowangle boundaries (LABs) were present in the interior of the $\mathrm{Cu}$ grains (as shown in Fig. 3a). As illustrated in Fig. 3(b), after the first-cycle REFF process, the elongated grains were nearly all equiaxed shapes, and the LABs were still dominant in the interior of grains (as shown in Fig. 4b). Moreover, the initial grain boundaries could be observed clearly as inverse pole figure (IPF) EBSD maps. However, after the second cycle of the REFF process, the fraction of HABs increased in the extruded and forged samples (as shown in Fig. 4(c), (d) and Table 1). The average grain size, the number of interfaces per unit test line length $P_{\mathrm{L}}$ and the stereology parameter $S_{\mathrm{V}}$ are displayed in Table 1. The grain size measurement in the $X$ and $Y$ directions, parallel and perpendicular to the axis (ED/FD), respectively, was carried out using the linear intercept method without taking the twin boundaries into account. The graindeformed matrix interface area density per unit volume $S_{\mathrm{V}}$ is (Ref 23):

$S_{\mathrm{V}}=P_{\mathrm{L}, X}+P_{\mathrm{L}, Y}$

$P_{\mathrm{L}}=N / L$

$d=L / N$

The grain-deformed matrix interface area density per unit volume can be obtained by substituting Eq 3 and 4 into Eq 2 as follows:

$S_{\mathrm{V}}=1 / d_{X}+1 / d_{Y}$

with the increasing strain, the recrystallization parameter $S_{\mathrm{V}}$ increased gradually as shown in Table 1 . This indicated directly that the fraction of HABs increased and grain refinement occurred. Refine equiaxed grains with an average grain size down to $0.8 \mu \mathrm{m}$ appeared alongside subgrains as shown in Fig. 2(d). The majority of the small equiaxed grains in Fig. 2(d) were the red grains, with a $<100>$ orientation, but most others had orientations $\langle 110\rangle$ and $\langle 111\rangle$ and were nearly maintained in the deformation matrix. Most of the initial grain boundaries had become fuzzier and curved compared to the sample fabricated by the first cyclic of the REFF process. This phenomenon was very similar to those found in pure copper and aluminum after tension deformed (Ref 24, 25). It was found that equiaxed cell structures and dense dislocation wall structures were observed in grains of different orientations in a polycrystal metal. For grains oriented near the [100], it appeared that the formation of these structures may be related to the fact that four-to-eight slip systems always have high and similar Schmid factors. However, the deformation matrix 


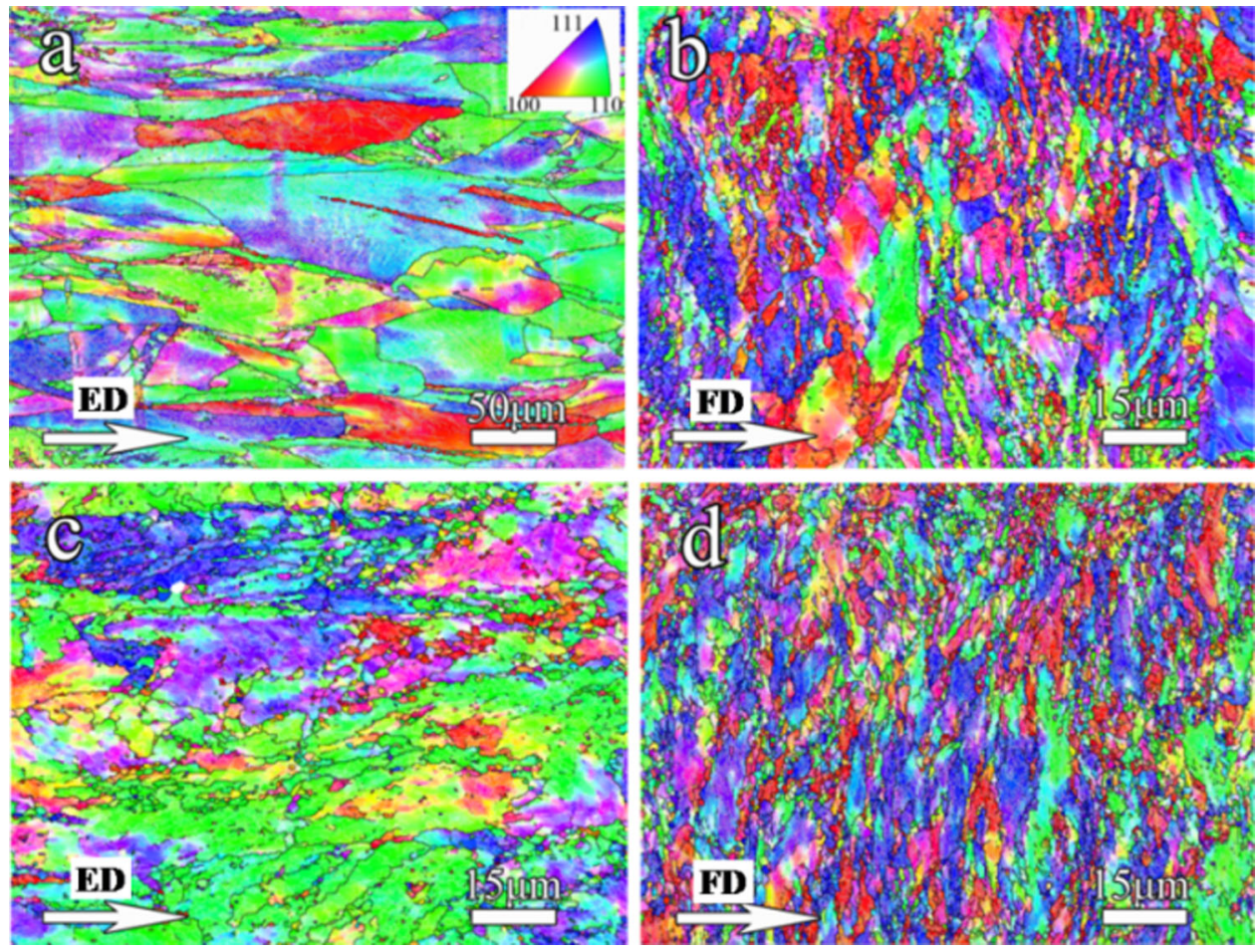

Fig. 3 Inverse pole figure EBSD maps after one cycle of the REFF process: (a) after extrusion and (b) after free forging, and two cycles of the REFF process: (c) after extrusion and (d) after free forging
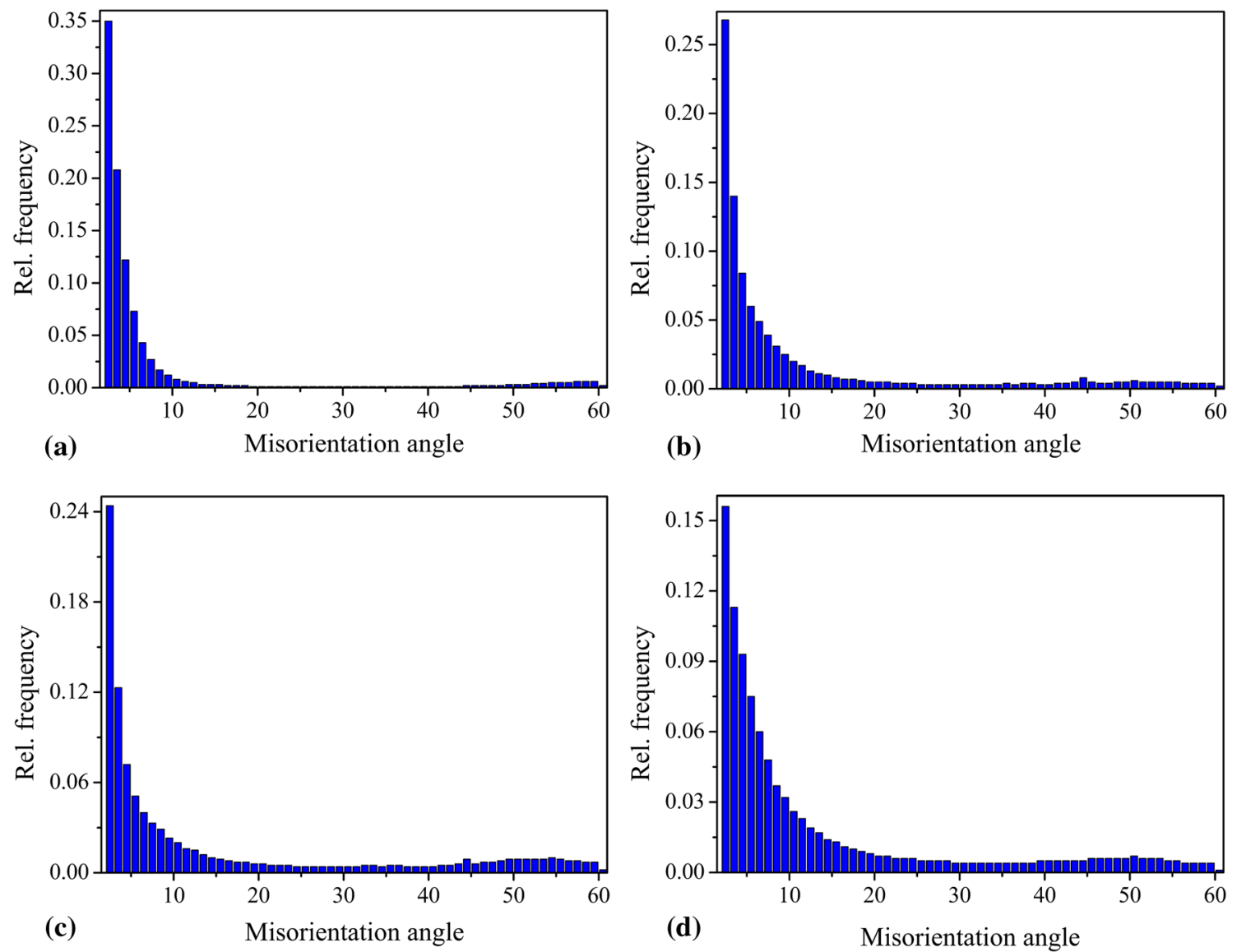

Fig. 4 Misorientation angle distributions after one cycle of the REFF process: (a) after extrusion and (b) after free forging, and two cycles of the REFF process: (c) after extrusion and (d) after free forging 
structures were always on the primary slip plane (11-1), which led to grains oriented near the [100] form predominantly an equiaxed structure, while other grains formed dense dislocation wall structures more easily.

The main mechanism of grain refinement using REFF was the elongation of grains during extrusion, which were fragmented by the maximum tangential stress that was produced by shearing on the slip lines during free forging (Ref 21). During a cycle of the REFF process, as shown in Fig. 3 and 4, in order to match the plastic strain produced by SPD, a suite of slip systems were activated in the interior of grains, which led to a large amount of LABs and dislocation boundaries. The generation of new boundaries, including LABs and dislocation boundaries inside a grain could be interaction with the increase in strain. After two passes of the REFF process, the elongated structure in the extruded copper billet, as shown in Fig. 3(a), was replaced by a structure predominantly consisting of free forging billets with small equiaxed grains (as displayed in Fig. 3d). With an increase in the strain, the density of dislocation continued to increase and the LABs absorbed dislocation and increased their misorientation due to rise in the fraction of HABs and the formation of new finer grains, leading to grain refinement (Ref 26). However, the dislocation activities in the interior of finer grains became more difficult in this condition. Subgrain rotation, leading to an increase in the misorientation and an increase in the fraction of HAGs and grain refinement, occurs to accommodate such plastic deformation. Otherwise, the $\langle 100\rangle$ orientation grains became subdivided by ordinary dislocation cell boundaries defining a three-dimensional cell structure (Ref 27). This orientation of grains would lead to more homogenous grain size compared to others because of the dynamic recrystallization.

The evolution of the crystallographic texture and the volume fractions of the fiber textures, in the form of inverse pole figures of the longitudinal direction (parallel to ED or FD) samples axis, are displayed in Fig. 5. The fully annealed CG copper samples have a relatively weak texture, with mixed $<100>+<111>$ fibers parallel to the longitudinal direction.

Table 1 Average grain size, PL (in $X$ and $Y$ direction) and $S_{V}$ from EBSD analysis of the initial CG copper and post-REFF copper

\begin{tabular}{lccllll}
\hline & \multicolumn{2}{c}{ Average grain size, $\boldsymbol{\mu m}$} & & & \\
\cline { 2 - 3 } & $\boldsymbol{X}$ direction & $\boldsymbol{Y}$ direction & & $\boldsymbol{P}_{\mathbf{L}, \boldsymbol{X}}$ & $\boldsymbol{P}_{\mathbf{L}, \boldsymbol{Y}}$ & $\boldsymbol{S}_{\mathbf{V}}$ \\
\hline Initial & $44.1 \pm 4$ & $37.6 \pm 3$ & 0.023 & 0.027 & 0.05 \\
First extrusion & $25.4 \pm 1.3$ & $10.7 \pm 1$ & 0.039 & 0.093 & 0.13 \\
First forging & $2.6 \pm 0.5$ & $3.5 \pm 0.5$ & 0.38 & 0.29 & 0.67 \\
Second extrusion & $2.5 \pm 0.15$ & $2.3 \pm 0.2$ & 0.4 & 0.43 & 0.83 \\
Second forging & $0.8 \pm 0.08$ & $0.9 \pm 0.06$ & 1.25 & 1.11 & 2.36 \\
\hline
\end{tabular}

After the first cycle of the REFF process, a different fiber texture was obtained. Through the extrusion pass, the $<111>$ fiber was strengthened significantly, and the $\langle 100\rangle$ fiber texture was nearly absent. However, the $\langle 111\rangle$ texture produced during the extrusion zone completely disappeared by the following free forging process, giving rise to the $<110>$ fiber texture. At later cycles of the REFF process, the $<111>$ texture and the $\langle 110\rangle$ texture alternately appeared in the UFG pure copper samples. Compared to the first-cycle samples, the $<111>$ fiber texture produced during extrusion process was evidently weakened and the $<100\rangle$ texture was relative stabilized low value data after the second extrusion. However, the $<110>$ texture generated during free forging process was significantly strengthened.

The casting texture during a continuous unidirectional solidification process largely depends on the crystal structure of the material. It is known that the preferential growth orientation of face-centered cubic (fcc) copper is $<100>$ (Ref 27). As can be seen in Fig. 5, the coarse-grained copper mainly grew along the $<100\rangle$ direction. After the REFF process, because the $<111>$ fiber is known as the extrusion texture of fcc metals, and the $\langle 110\rangle$ fiber texture is the characteristic for compression texture (Ref 22), the production of $<111>$ fiber during the extrusion process was very evidently reduced following the free forging process, giving rise to the $<110>$ fiber. The opposite texture evolution produced in the extruded and forged sample was due to the complete inversion of the strain of the extrusion and free forging process. Therefore, the $<111>$ fiber texture generated during the extrusion process was replaced by the $<110>$ fiber texture produced during the

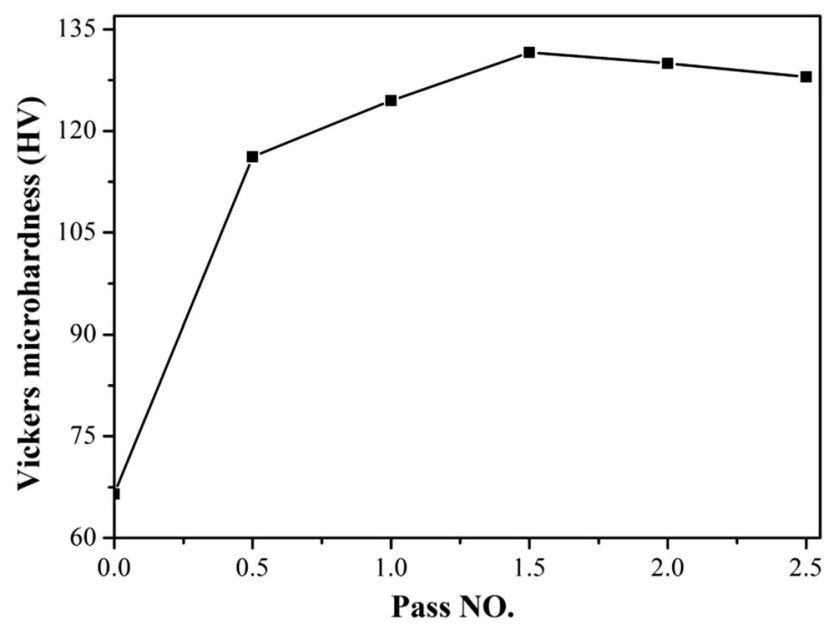

Fig. 6 Microhardness as a function of pass number in the REFF process. $(0.5,1.5$ and 2.5 represent the extrusion process)
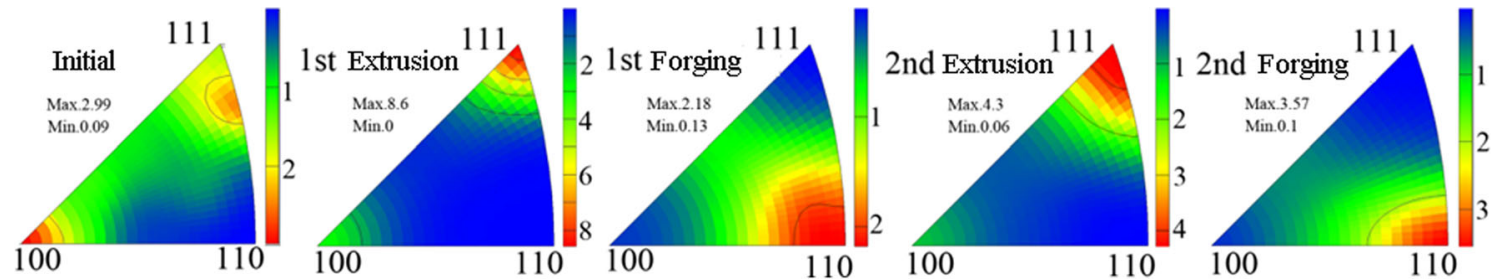

Fig. 5 The evolution of the fiber texture in the inverse pole of the longitudinal direction (ED/FD) sample during REFF 
forging process. Otherwise, the interaction of the opposite texture components led to a weakened texture UFG copper billet for post-forging.

\subsection{Mechanical Behavior of Extruded and Free Forged Copper}

The results of microhardness tests for the extruded and free forged specimens are displayed in Fig. 6. After the first extrusion process, the microhardness of the fully annealed copper increased sharply from 63 to $114 \mathrm{HV}$. This was due to an

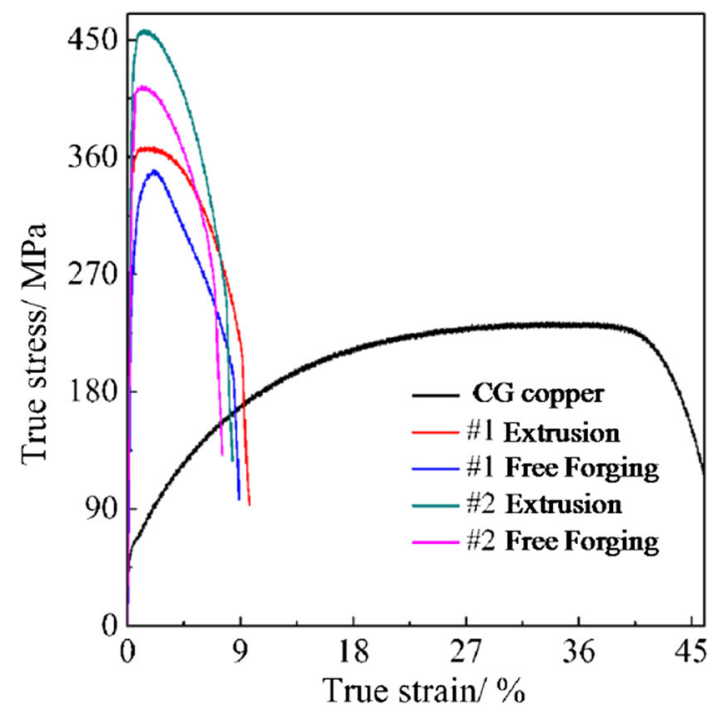

Fig. 7 True stress-strain curves under tension along the LD of the copper billets increase in the work hardness. However, at the end of the first REFF cycle, the hardness exhibited only a slight increase, from 114 to $122 \mathrm{HV}$. A maximum value of hardness $131 \mathrm{HV}$ was observed at the end of the second extrusion process. Some possible mechanisms of the improvement in hardness of the SPD copper fabricated by the REFF process from CG copper are discussed below. For CG pure copper, the stereology parameter $S_{\mathrm{V}}$ was smaller than SPD samples as shown in Table 1. Therefore, the volume density of high-angle grain boundary, a barrier for dislocations motion, increased with increasing strain. The CG copper structures had a weak resistance to the dislocation motion, and slip system could move easily, resulting in the softening of the microhardness. After the cyclic REFF process, several grain fragmentation and orientation led to more defects. An increase in the density of dislocations and HABs (as shown in Fig. 4) during SPD, resulting in the dislocation movement being resisted in the hardness test, led to an increase in microhardness. Finally, the hardness was slightly reduced after the end of the third cycle of the REFF process. This was due to dynamic recovery and recrystallization.

Figure 7 and Table 2 show the true stress-strain curves of the copper fabricated by the REFF process. The CG sample showed high ductility and low yield strength. After the REFF process, the ultimate tensile strength (UTS) and yield strength (YS) of all samples were remarkably increased. The UTS and YS values of the copper after two cycles REFF process were 451 and $462 \mathrm{MPa}$, respectively. The samples retained a sufficient ductility of $8.3 \%$. The REFF process combines two strengthening mechanisms: grain boundary strengthening and dislocation strengthening. In fact, increasing dislocation contributed to the improvement in the tensile strength. Moreover, the fine grains resulting from the REFF process helped to maintain a sufficient ductility even after a significant straining

Table 2 The yield strengths, ultimate strengths and elongations due to fracture under tension along the longitudinal direction

\begin{tabular}{lccr}
\hline Samples & Yield strength, MPa & Ultimate strength, MPa & Elongation, \% \\
\hline Initial annealed & $79 \pm 2$ & $241 \pm 1$ & $42.5 \pm 3$ \\
First extrusion & $334 \pm 4$ & $345 \pm 2$ & $8.8 \pm 1$ \\
First forging & $361 \pm 3$ & $370 \pm 4$ & $9.4 \pm 1$ \\
Second extrusion & $407 \pm 4$ & $415 \pm 2$ & $7.9 \pm 0.8$ \\
Second forging & $451 \pm 2$ & $462 \pm 1$ & $8.3 \pm 1.3$ \\
\hline
\end{tabular}
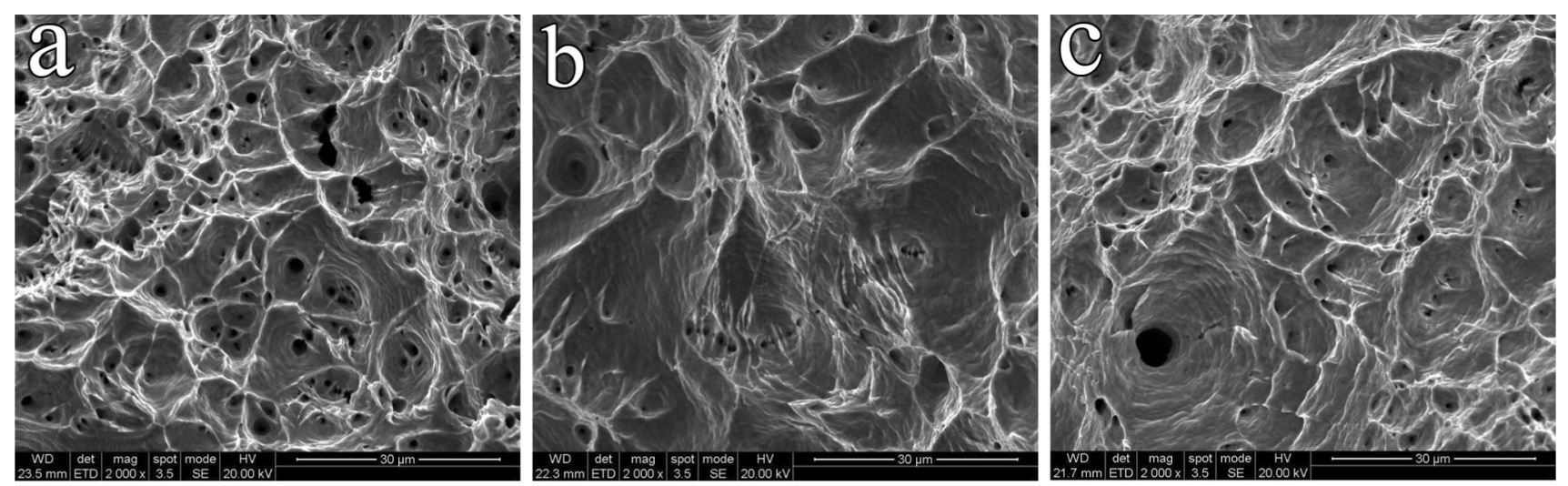

Fig. 8 SEM fractograph of (a) CG copper, (b) second extrusion and (c) second free forging 
from the cold REFF processing. On the other hand, the increase in the elongation in the extrusion billets, compared with the free forged ones, was due to a lower decrease in the work hardening rate when the stress increased. In order to study the pure copper billets possessing fast necking behavior after each REEF process, the fracture surface was evaluated using SEM. These results are shown in Fig. 8. It was obvious that a relatively flat zone could be observed adjacent to a dimple zone, which was the typical fracture characteristic for $\mathrm{Cu}$. However, compared with CG copper, the fracture surface in the second cycle REEF samples mainly consisted of dimples with a number of cleavage planes that exhibit typical toughness fracture character. This led to sufficient ductility and a fast necking phenomenon.

\section{Conclusion}

In the present study, copper billets for mass applications were successfully and efficiently fabricated by REFF, without using any additional tools or devices. Several conclusions were reached as follows:

1. After two cycles of the REFF process, the sum effective strain was $\varepsilon=4.7$. The average grain size was measured to be $0.8 \mu \mathrm{m}$.

2. The $\langle 111\rangle$ texture and $\langle 110\rangle$ texture alternately appeared in the pure copper samples during the REFF process. This was beneficial for obtaining a weakened texture microstructure in the $\mathrm{Cu}$ billets.

3. The yield strength of samples was remarkably increased to $462 \mathrm{MPa}$ after two cycles of the REFF process, and the samples retained a sufficient ductility of $8.3 \%$.

\section{Acknowledgments}

This research is funded by Chongqing Research Program of Basic Research and Frontier Technology (No. cstc2015jcyjBX0115) and Guangdong Academy of Science Fund (2018GDASCX-0967).

\section{Open Access}

This article is distributed under the terms of the Creative Commons Attribution 4.0 International License (http://creativecommons.org/ licenses/by/4.0/), which permits unrestricted use, distribution, and reproduction in any medium, provided you give appropriate credit to the original author(s) and the source, provide a link to the Creative Commons license, and indicate if changes were made.

\section{References}

1. J. Chen, X. Ma, W. Yan, F. Xia, and X. Fan, Effect of Transverse Grain Boundary on Microstructure, Texture and Mechanical Properties of Drawn Copper Wires, J. Mater. Sci. Technol., 2014, 30, p 184-191

2. L. Peng, H. Xie, G. Huang, Y. Li, X. Yin, X. Feng, X. Mi, and Z. Yang, The Phase Transformation and Its Effects on Properties of a $\mathrm{Cu}$ $0.12 \mathrm{wt} \%$ Zr Alloy, Mater. Sci. Eng. A, 2015, 633, p 28-34

3. C. Zhu, A. Ma, J. Jiang, X. Li, D. Song, D. Yang, Y. Yuan, and J. Chen, Effect of ECAP Combined Cold Working on Mechanical Properties and Electrical Conductivity of Conform-Produced Cu-Mg Alloys, $J$. Alloys Compd., 2014, 582, p 135-140

4. W. Luo, H. Gong, Q. Wang, J. Lu, and M. Yan, Effect of Cryogenic Impact Deformation and Subsequent Annealing on Microstructure and Microhardness of Pure Copper, Mater. Lett., 2015, 157, p 315-317

5. T. Hartwig, Grain Refinement and Recrystallization of Heavily Worked Tantalum, Mater. Sci. Eng. A, 2006, 426, p 128-142

6. O. Saray, G. Purcek, I. Karaman, and H.J. Maier, Improvement of Formability of Ultrafine-Grained Materials by Post-SPD Annealing, Mater. Sci. Eng. A, 2014, 619, p 119-128

7. R.A.G. Silva, A. Paganotti, A.T. Adorno, C.M.A. Santos, and T.M. Carvalho, Precipitation Hardening in the Cu-11wt.\%Al-10wt.\%Mn Alloy with Ag Addition, J. Alloys Compd., 2015, 643, p S178-S181

8. R.F. Zhu, J.N. Liu, G.Y. Tang, S.Q. Shi, and M.W. Fu, Properties, Microstructure and Texture Evolution of Cold Rolled $\mathrm{Cu}$ Strips Under Electropulsing Treatment, J. Alloys Compd., 2012, 544, p 203-208

9. X. Sauvage, P. Jessner, F. Vurpillot, and R. Pippan, Nanostructure and Properties of a $\mathrm{Cu}-\mathrm{Cr}$ Composite Processed by Severe Plastic Deformation, Scr. Mater, 2008, 58, p 1125-1128

10. M.Z. Bian, Y.L. Li, M. Mathesh, D. Abreu, and N.D. Nam, Microstructure and Texture Evolutions and Mechanical Properties in Pure Copper by Equal-Channel Angular Pressing, J. Alloys. Compd., 2013, 578, p 369-372

11. Y. Estrin and A. Vinogradov, Extreme Grain Refinement by Severe Plastic Deformation: A Wealth of Challenging Science, Acta Mater., 2013, 61, p 782-817

12. S. Lu, Q.M. Hu, E.K. Delczeg-Czirjak, B. Johansson, and L. Vitos, Determining the Minimum Grain Size in Severe Plastic Deformation Process via First-Principles Calculations, Acta Mater, 2012, 60, p 4506-4513

13. M.J. Starink, X.G. Qiao, J. Zhang, and N. Gao, Predicting Grain Refinement by Cold Severe Plastic Deformation in Alloys Using Volume Averaged Dislocation Generation, Acta Mater, 2009, 57, p 5796-5811

14. Y.Z. Tian, S.D. Wu, Z.F. Zhang, R.B. Figueiredo, N. Gao, and T.G. Langdon, Comparison of Microstructures and Mechanical Properties of a Cu-Ag Alloy Processed Using Different Severe Plastic Deformation Modes, Mater. Sci. Eng. A, 2011, 528, p 4331-4336

15. M. Wang, R.S. Averback, P. Bellon, and S. Dillon, Chemical Mixing and Self-Organization of $\mathrm{Nb}$ Precipitates in $\mathrm{Cu}$ During Severe Plastic Deformation, Acta Mater., 2014, 62, p 276-285

16. K.X. Wei, W. Wei, F. Wang, Q.B. Du, Alexandrov, IV., and J. Hu, Microstructure, Mechanical Properties and Electrical Conductivity of Industrial $\mathrm{Cu}-0.5 \% \mathrm{Cr}$ Alloy Processed by Severe Plastic Deformation, Mater. Sci. Eng. A, 2011, 528, p 1478-1484

17. M.H. Farshidia, M. Kazeminezhad, and H. Miyamoto, Microstructure and Mechanical Properties of an Al-Mg-Si Tube Processed by Severe Plastic Deformation and Subsequent Annealing, Mater. Sci. Eng. A, 2015,640 , p $42-50$

18. N. Maury, N.X. Zhang, Y. Huang, A.P. Zhilyaev, and T.G. Langdon, A Critical Examination of Pure Tantalum Processed by High-Pressure Torsion, Mater. Sci. Eng. A, 2015, 638, p 174-182

19. Z. Lin, L. Wang, X. Xue, W. Lu, J. Qin, and D. Zhang, Microstructure Evolution and Mechanical Properties of a Ti-35Nb-3Zr-2Ta Biomedical Alloy Processed by Equal Channel Angular Pressing (ECAP), Mater. Sci. Eng. C Mater. Biol. Appl., 2013, 33, p 4551-4561

20. R. Ding, C. Guo, and S. Guo, Assessment of Anisotropic Tensile Strength Using a Modified Shear Punch Test for Cu-Cr-Zr Alloy Processed by Severe Plastic Deformation, Mater. Sci. Eng. A, 2013, 587, p 320-327

21. L. Zaharia, R. Comaneci, R. Chelariu, and D. Luca, A New Severe Plastic Deformation Method by Repetitive Extrusion and Upsetting, Mater. Sci. Eng. A, 2014, 595, p 135-142

22. N. Pardis, C. Chen, R. Ebrahimi, L.S. Toth, C.F. Gu, B. Beausir, and L. Kommel, Microstructure, Texture and Mechanical Properties of Cyclic Expansion-Extrusion Deformed Pure Copper, Mater. Sci. Eng. A, 2015, 628, p 423-432

23. W. Guilin and D.J. Jensen, Automatic Determination of Recrystallization Parameters Based on EBSD Mapping, Mater. Chanract., 2008, 59, p 794-800

24. X. Huang, Grain Orientation Effect on Microstructure in Tensile Strained Copper, Scr. Mater., 1998, 38(11), p 1697-1703 
25. X. Huang and N. Hansen, Grain Orientation Dependence of Microstructure in Aluminium Deformed in Tension, Scr. Mater. 1997, 37(1), p 1-7

26. G. Yang, Z. Li, Y. Yuan, and Q. Lei, Microstructure, Mechanical Properties and Electrical Conductivity of $\mathrm{Cu}-0.3 \mathrm{Mg}-0.05 \mathrm{Ce}$ Alloy Processed by Equal Channel Angular Pressing and Subsequent Annealing, J. Alloys Compd., 2015, 640, p 347-354
27. X. Wang, X. Liu, and J. Xie, Mechanism of Surface Texture Evolution in Pure Copper Strips Subjected to Double Rolling, Prog. Nat. Sci. Mater. Int., 2014, 24, p 75-82

Publisher's Note Springer Nature remains neutral with regard to jurisdictional claims in published maps and institutional affiliations. 